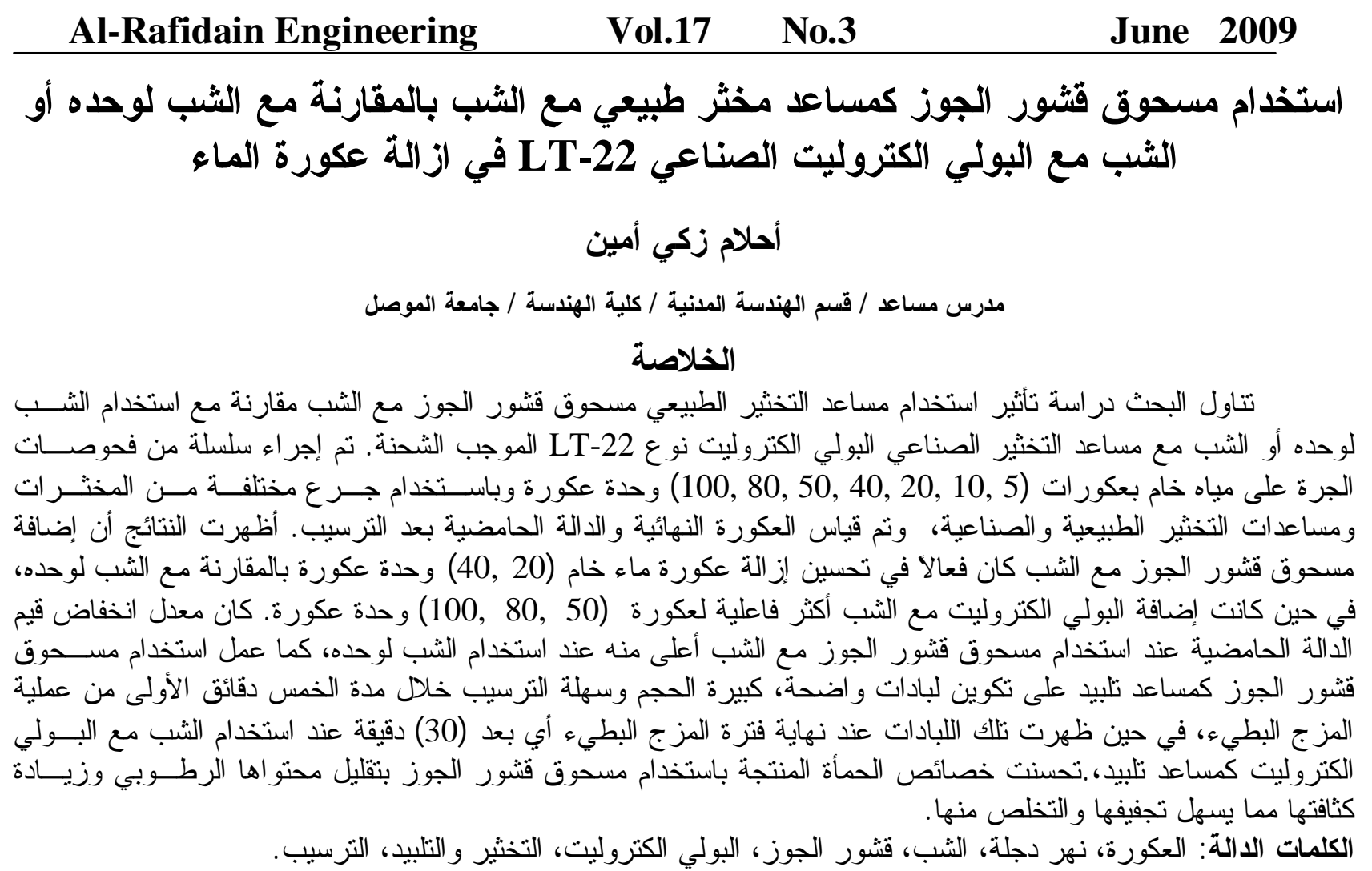

\title{
Use of Walnut (Juglans SPP.) Coat as Natural Coagulant Aid with Alum Compared with Alum Alone or Alum with Synthetic Polyelectrolyte in Turbidity Removal
}

\author{
Ahlam Zeki Ameen \\ Ass. Lecturer - college of Engg. University of Mosul
}

\begin{abstract}
This research includes studying the effect of using natural coagulant aid "Walnut Coat" with alum and synthetic polyelectrolyte with alum also on turbidity removal compared with alum alone. A set of jar test was conducted on raw water of different turbidities $(5,10,20,40,50,80$ and 100) NTU using different doses of coagulants and coagulant aids. Final turbidity, pH., were measured after settling. The obtained results indicated that the use of walnut coat with alum was effective in the removal of turbidities of $(20,40)$ NTU compared with alum alone. While the addition of polyelectrolyte with alum was found to be more effective for higher turbidities $(50,80,100)$ NTU. Additionally, the average reduction in $\mathrm{pH}$-value was more using walnut coat than that of using alum alone. Furthermore, walnut coat led to the formation of large, clear and easily settled flocs within the first 5 minutes of slow mixing, while same flocs appears at the end of 30 minutes of slow mixing with alum and polyelectrolyte as flocculant aid. Better sludge characteristics were obtained with walnut coat as flocculant aid, with low water content and high density which can be easily dried and disposed.
\end{abstract}

Key words: Turbidity, Tigris River, Alum, Walnut Coat, Polyelectrolyte, Coagulation, flocculation, Sedimentation. 


\section{المقدمة ومر اجعة المصادر:}

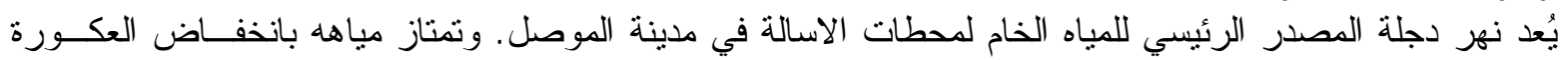

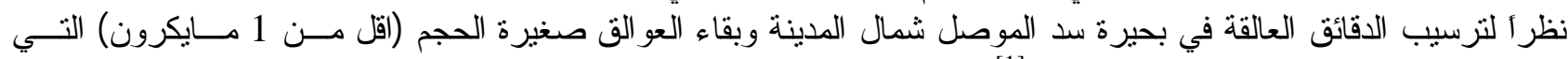

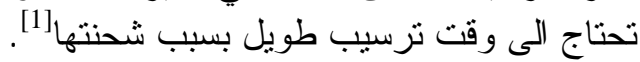

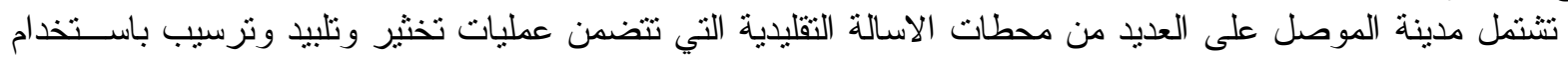

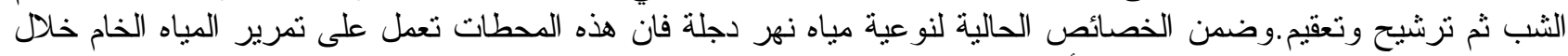

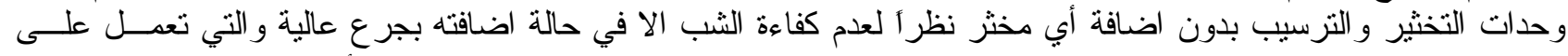

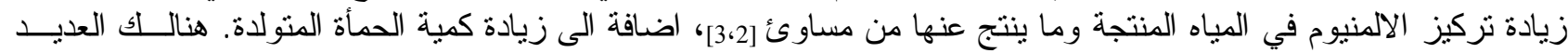

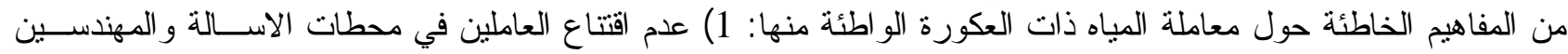

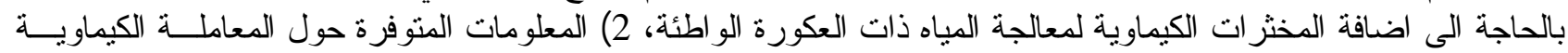

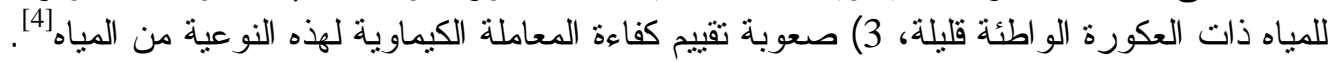

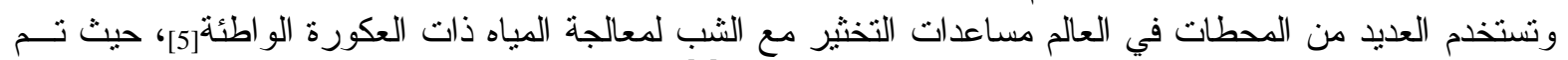

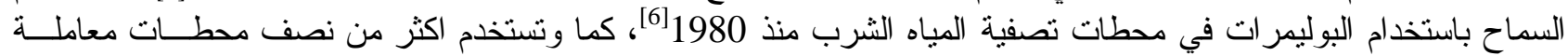

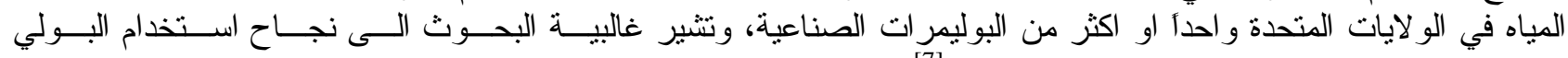

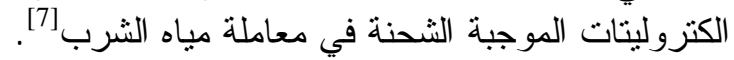
و هناللك العديد من الدر اسات حول مساعدات التخثيرحيث درس Muyibi S, و واخرون[8] 2003 كفاءة خلاصة بـذأور

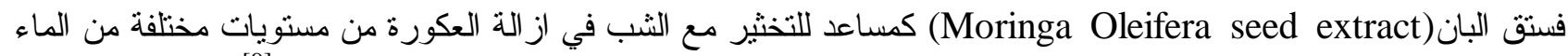

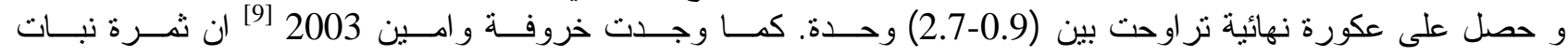

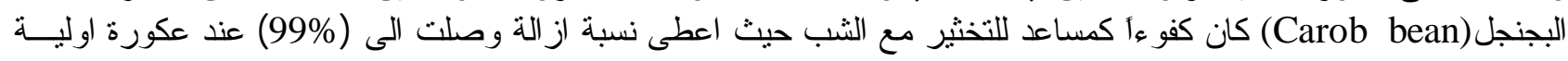

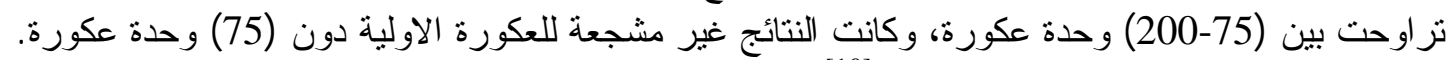

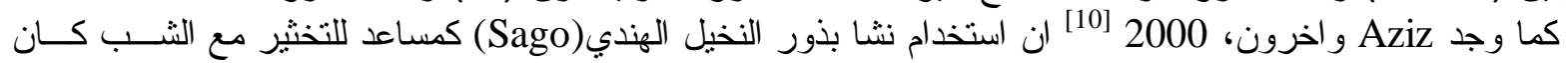

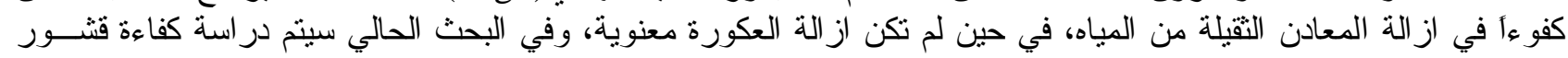

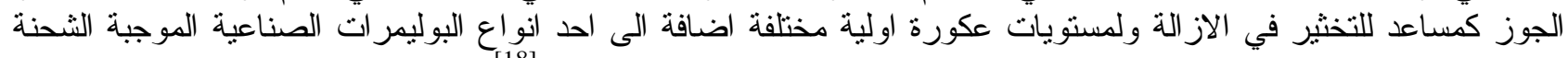

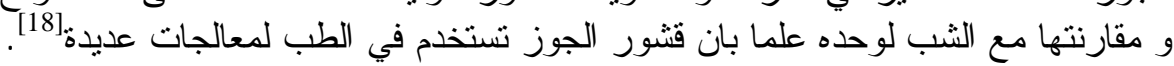

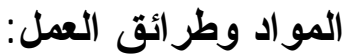

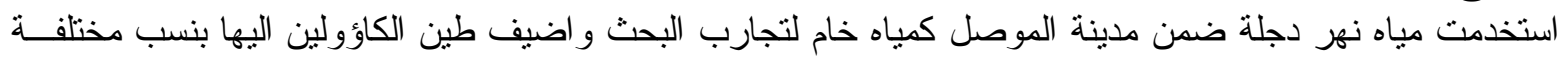

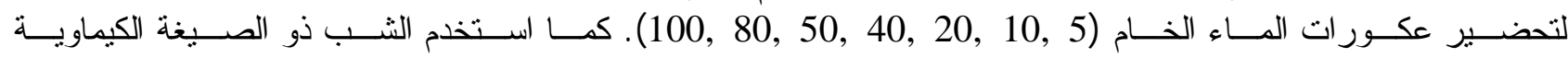
( $\left.\mathrm{Al}_{2}\left(\mathrm{SO}_{4}\right)_{3} \cdot 16 \mathrm{H}_{2} \mathrm{O}\right)$

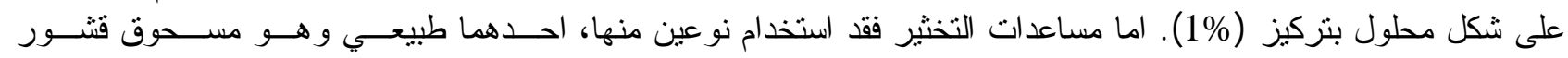

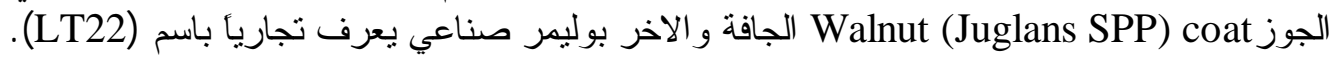

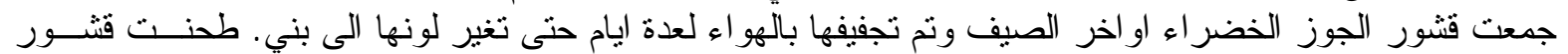

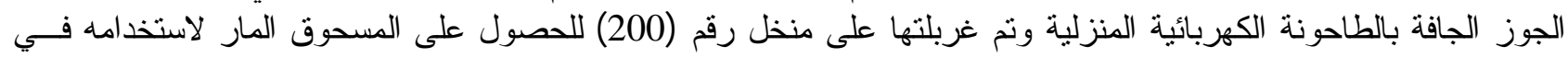

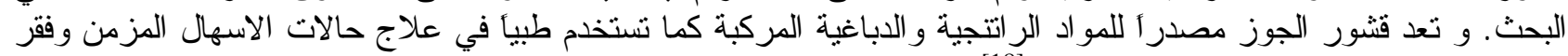

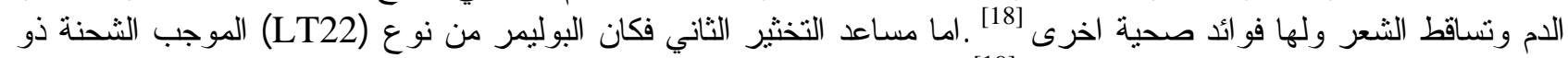

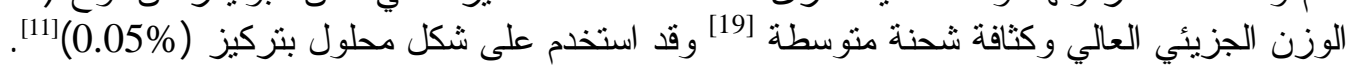

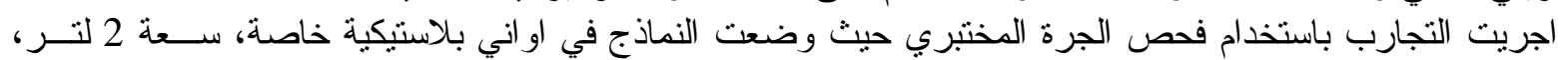

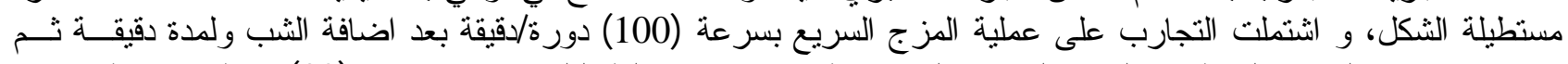

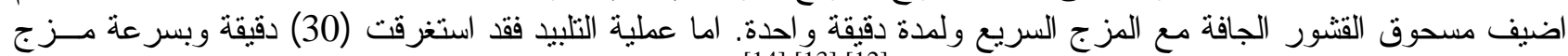

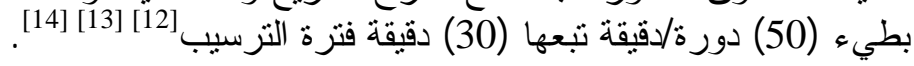

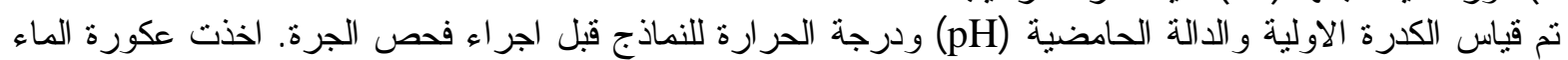

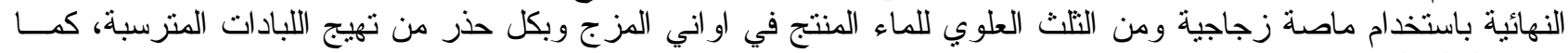
قيست الدالة الحامضية.

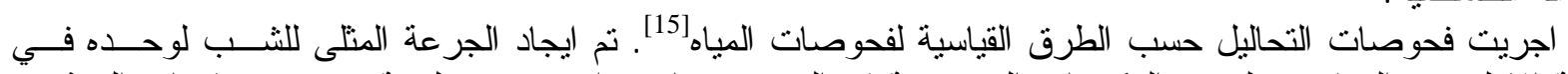

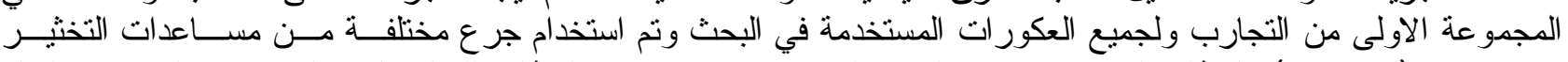

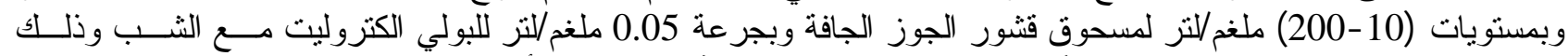

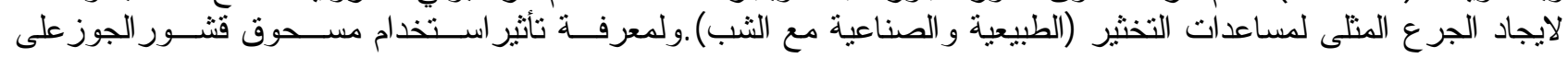


الخو اص الكيمياويه للمياه تم قياس العسره و التوصيل الكهربائي للماء الخام ولنماذج المياه بعد المعامله للجرع المتلى لمساعدات

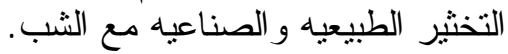

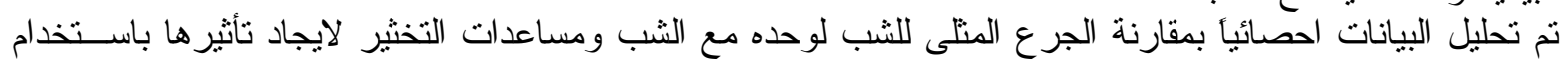

اختبار دنكن ولجميع مستويات العكورة.

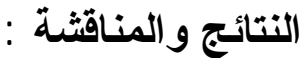

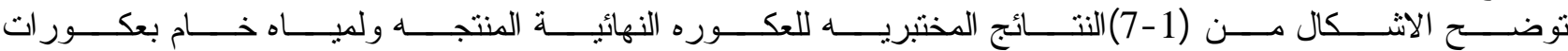

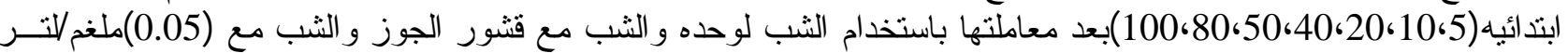

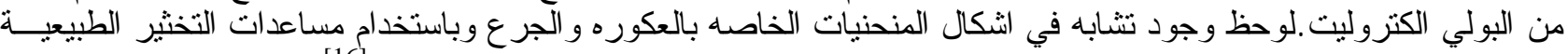

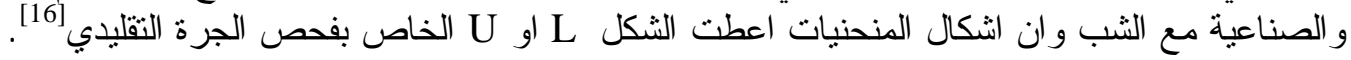

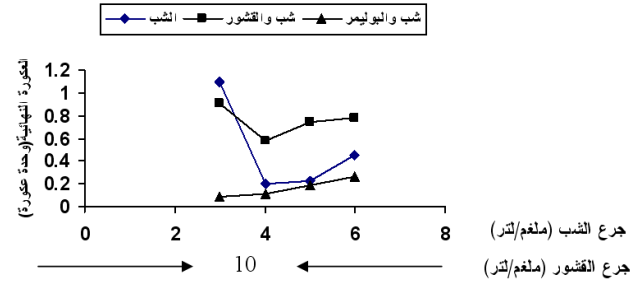

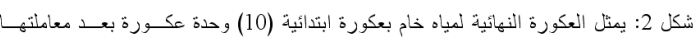

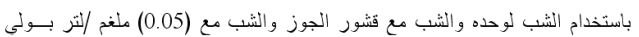

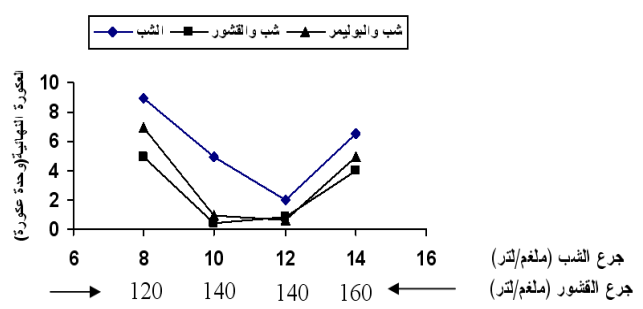

شكل 4: بمتل العكورة النهائية لمياه خام بعكو رة ابتدائية (40) وحدة عكورة بعد معاملتها باستخدام

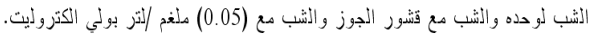

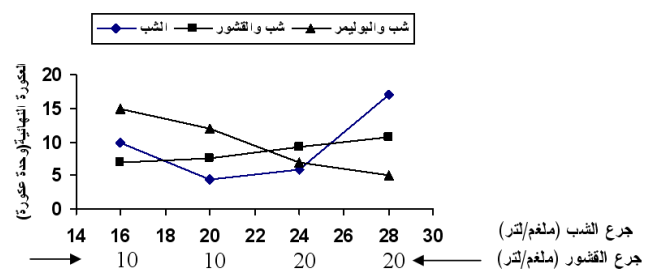

شكل 6: يمثلّ العكورة النهائية لمياه خام بعكورة ابتدائية (80) وحدة عكورة بعد معامنتها باستخدام

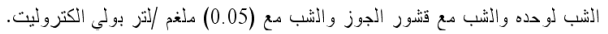

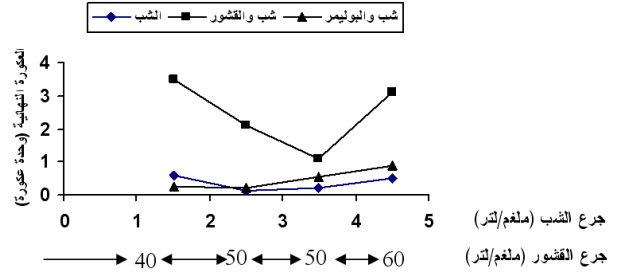

شكل 1: يمثل العكورة النهائبةدة لمياه خام بعكورة ابتدائية (5) وحدة عكورة بعد معاملتها باستخدام الشب لوحده والثب مع قشور الجوز و الثشب مع (0.05) ملغم الثر بولي الكتروليت.

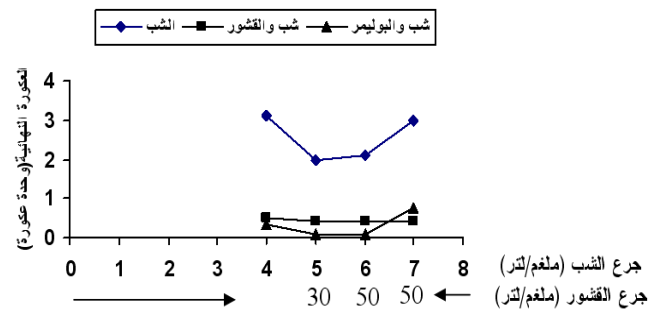

شكل 3: يمثل العكورة النهائية لمياه خام بعكورة ابتدائية (20) وحدة عكورة بعد معاملتها

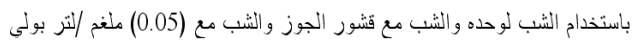

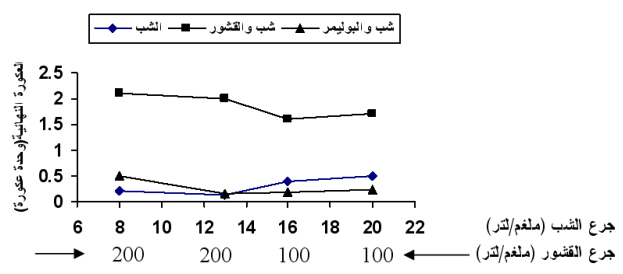

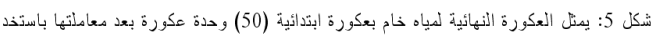

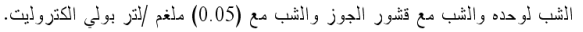

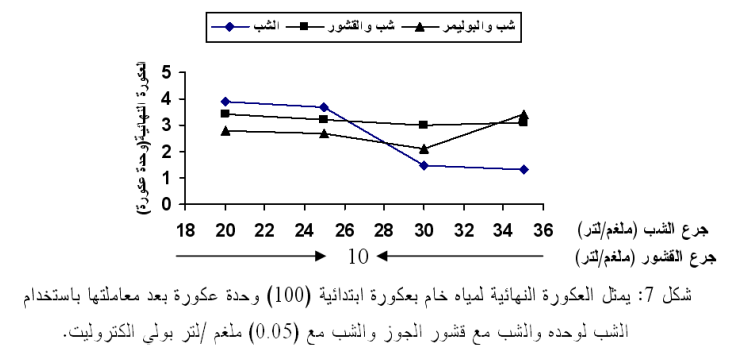


أمين :استخدام مسحوق قشور الجوز كمساعد مخثر طبيعي مع الثب بالمقارنة مع الثب لوحده أو الثب مع البولي

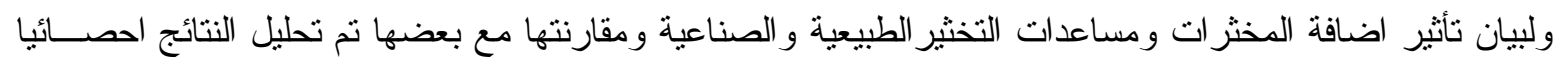

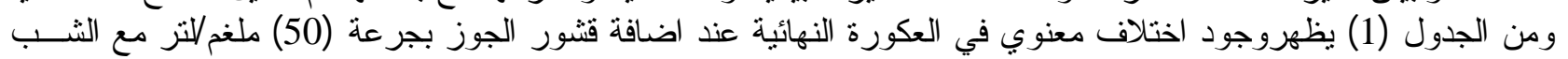

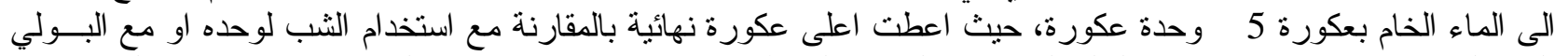

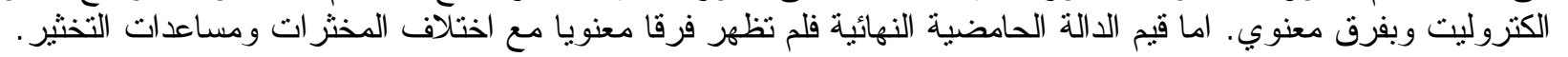

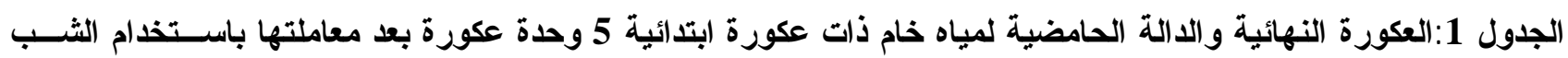

\begin{tabular}{|c|c|c|c|c|c|c|}
\hline \multirow{2}{*}{ مجموعة دنكن } & \multirow{2}{*}{$\begin{aligned} \text { المعدل الانحر اف } \\
\text { المعباري }\end{aligned}$} & \multirow{2}{*}{ دجموعة } & \multirow{2}{*}{ المعدل العدرة عكورة النهائية } & \multicolumn{3}{|c|}{ الجرعة (ملفم/لتر) } \\
\hline & & & & الكتروليتي & قشور الجوز & 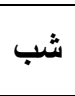 \\
\hline $\mathrm{A}$ & $0.06 \pm 7.09$ & A & $0.05 \pm 0.175$ & - & - & 2.5 \\
\hline $\mathrm{A}$ & $0.32 \pm 7.175$ & B & $0.63 \pm 1.65$ & - & 50 & 2.5 \\
\hline $\mathrm{A}$ & $0.16 \pm 7.26$ & $\mathrm{~A}$ & $0.04 \pm 0.23$ & 0.05 & - & 2.5 \\
\hline
\end{tabular}

P $\leq 0.05$ المعاملات ذات الحروف المختلفة عموديأ تعني وجود فرق معنوي عند مستوى

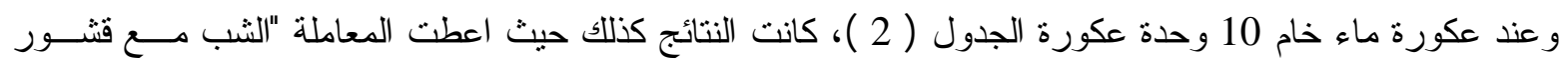

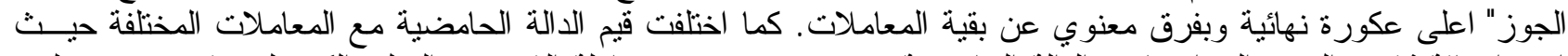

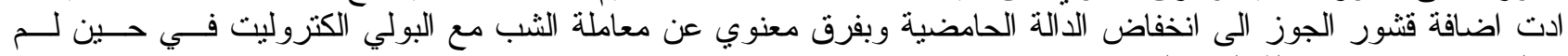

تختلف معنويا عن معاملة الثب لورن لوحده.

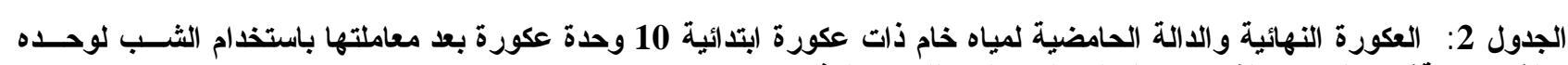

\begin{tabular}{|c|c|c|c|c|c|c|}
\hline \multirow{2}{*}{ دجموعة } & \multirow{2}{*}{$\begin{aligned} \text { المعدل الانحر افياري } \\
\end{aligned}$} & \multirow{2}{*}{ دجموعة } & \multirow{2}{*}{ العكورة النهائية وحدة ولمدرة } & \multicolumn{3}{|c|}{ الجرعة (ملفم/لتر) } \\
\hline & & & & الكتروليتي & قشوز & 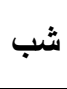 \\
\hline $\mathrm{AB}$ & $0.08 \pm 7.21$ & A & $0.02 \pm 0.215$ & - & - & 4 \\
\hline $\mathrm{B}$ & $0.11 \pm 6.82$ & $\mathrm{~B}$ & $0.12 \pm 0.665$ & - & 10 & 4 \\
\hline $\mathrm{A}$ & $0.21 \pm 7.45$ & $\mathrm{~A}$ & $0.014 \pm 0.10$ & 0.05 & - & 3 \\
\hline
\end{tabular}

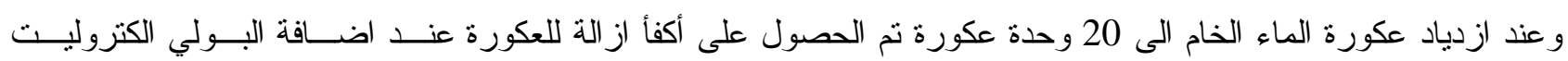

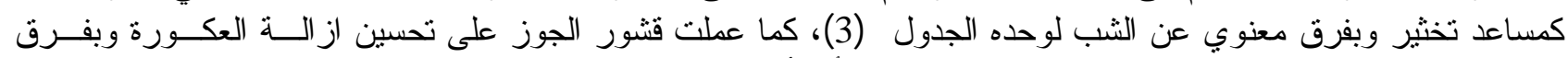

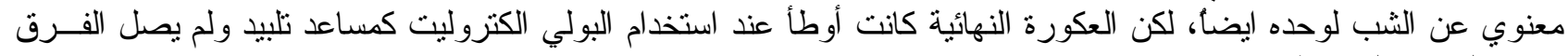
بينهما الى حد المعنوية.

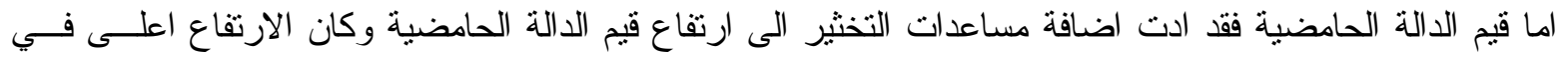

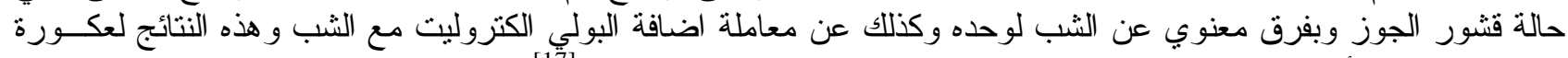

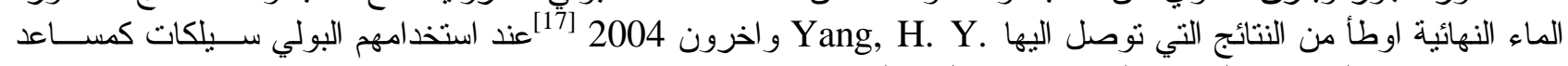

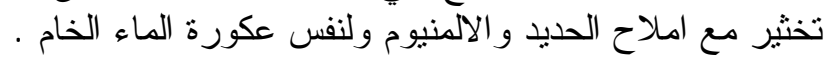

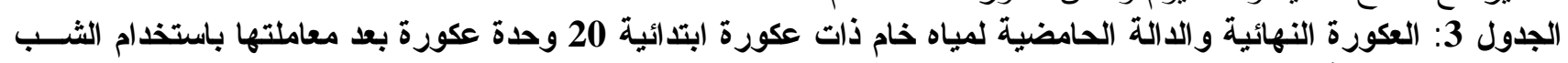

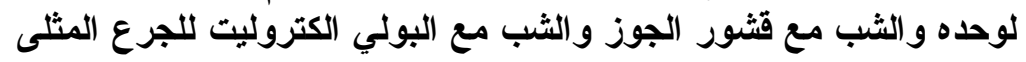

\begin{tabular}{|c|c|c|c|c|c|c|}
\hline \multirow{2}{*}{ مجموعة دنكن } & $\mathbf{p H}$ & \multirow{2}{*}{ مجموعة } & العكورة النهائية & \multicolumn{3}{|c|}{ الجرعة (ملفم/تتر) } \\
\hline & المعدل 土 الانحر اف المعياري & & المعدل 土 الاحسر اف & الكتروليتي & الجوز & شب \\
\hline $\mathrm{C}$ & $0.05 \pm 7.09$ & B & $0.07 \pm 2.05$ & - & - & 5 \\
\hline $\mathrm{A}$ & $0.13 \pm 7.995$ & $\mathrm{~A}$ & $0.05 \pm 0.465$ & - & 30 & 4 \\
\hline $\mathrm{B}$ & $0.07 \pm 7.40$ & $\mathrm{~A}$ & $0.205 \pm 0.18$ & 0.05 & - & 5 \\
\hline
\end{tabular}

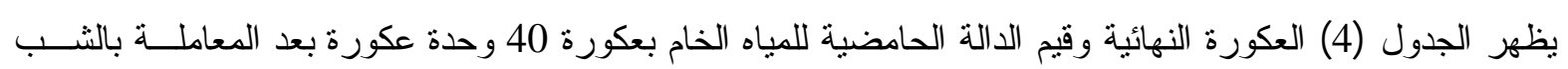

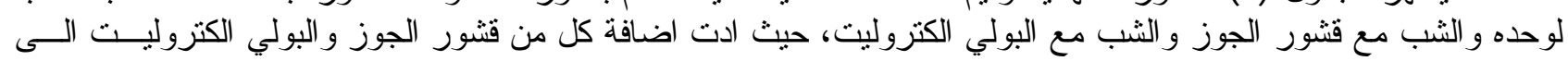

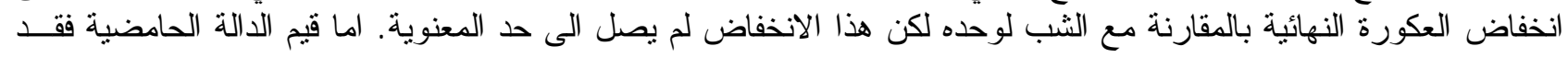




\section{Al-Rafidain Engineering $\quad$ Vol.17 $\quad$ No.3 $\quad$ June 2009}

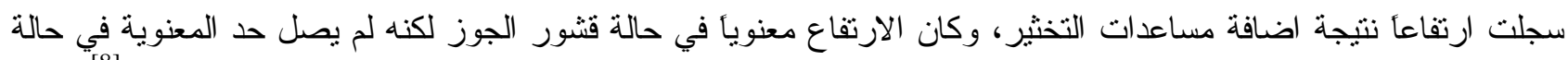

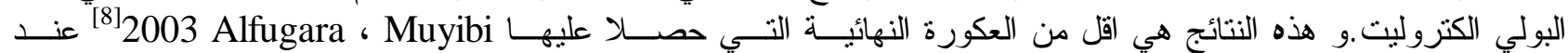

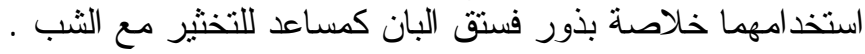

الجدول 4: العكورة النهائية والدالة الحامضية لمياه خام ذات عكورة ابتدائية 40 وحدة عكورة بعد معاملتها باستخدام الثــب

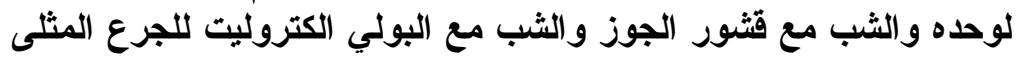

\begin{tabular}{|c|c|c|c|c|c|c|}
\hline \multirow{2}{*}{ مجموعة دنكن } & \multirow{2}{*}{ 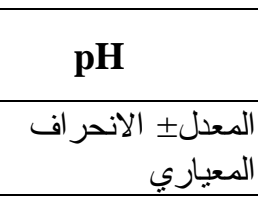 } & \multirow{2}{*}{ دجموعة } & ولعكورة النهائية & \multicolumn{3}{|c|}{ الجرعة (ملفم/لتر) } \\
\hline & & & المعدل الاحر اف المعياري & الكتروليتي & قثشوز & 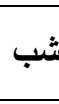 \\
\hline B & $0.15 \pm 6.55$ & A & $2.12 \pm 3.5$ & - & - & 12 \\
\hline A & $0.21 \pm 7.55$ & A & $0.28 \pm 0.60$ & - & 140 & 10 \\
\hline $\mathrm{B}$ & $0.09 \pm 6.69$ & $\mathrm{~A}$ & $0.21 \pm 0.75$ & 0.05 & - & 10 \\
\hline
\end{tabular}

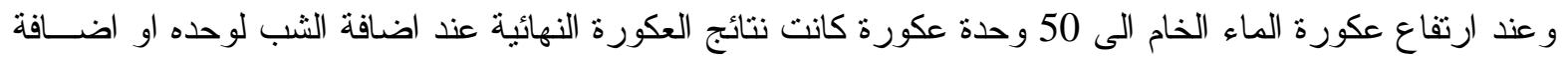

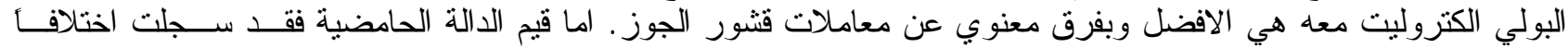

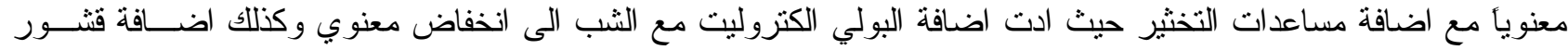

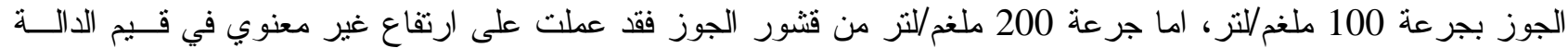

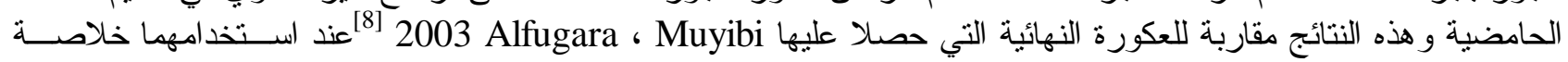
بذور فستق البان كمساعد تخثير مع الثب.

الجدول 5: العكورة النهائية والدالة الحامضية لميـاه خام ذات عكورة ابتدائية 50 وحدة عكورة بعد معاملتها باستخدام الثب

\begin{tabular}{|c|c|c|c|c|c|c|}
\hline \multirow{2}{*}{ مجموعة دنكن } & \multirow{2}{*}{ 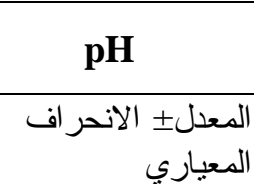 } & \multirow{2}{*}{ دجموعة } & \multirow{2}{*}{ 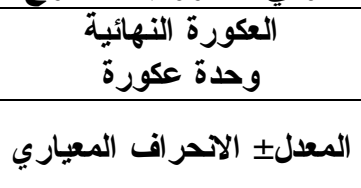 } & \multicolumn{3}{|c|}{ الجرعة (ملفم/لتر) } \\
\hline & & & & الكتروليتي & قشور الجوز & شب \\
\hline A & $0.11 \pm 6.38$ & A & $0.04 \pm 0.17$ & - & - & 13 \\
\hline B & $0.11 \pm 6.09$ & B & $0.07 \pm 1.65$ & - & 100 & 16 \\
\hline $\mathrm{B}$ & $0 \pm 6.75$ & $\mathrm{C}$ & $0.07 \pm 2.15$ & - & 200 & 8 \\
\hline $\mathrm{A}$ & $0.09 \pm 6.145$ & $\mathrm{~A}$ & $0.0 .05 \pm 0.205$ & 0.05 & - & 16 \\
\hline
\end{tabular}

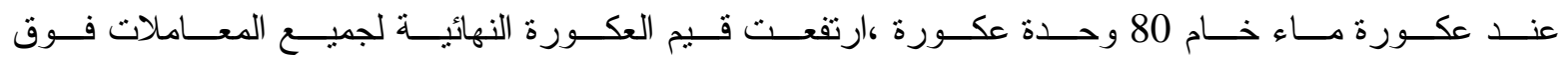

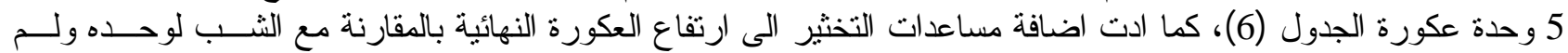

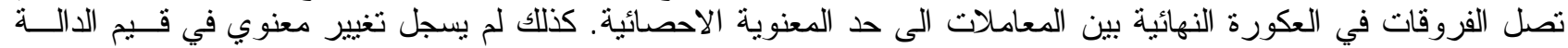

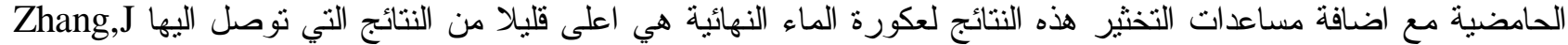

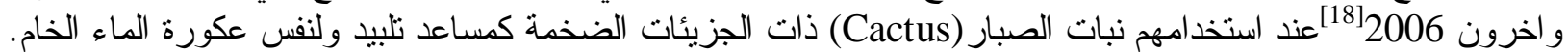

الجدول 6: العكورة النهائية و الا الة الحامضية لمياه خام ذات عكورة ابتدائية 80 وحدة عكورة بعد معاملتها باســتخدام الثــب

\begin{tabular}{|c|c|c|c|c|c|c|}
\hline \multirow{2}{*}{ مجموعة دنكن } & \multirow{2}{*}{$\begin{array}{r}\text { المعدل المعياري الانحر اف } \\
\text { المعلي }\end{array}$} & \multirow{2}{*}{ دجموعة } & \multirow{2}{*}{ 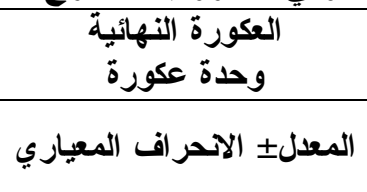 } & \multicolumn{3}{|c|}{ الجرعة (ملف/لتر) } \\
\hline & & & & الكتروليتي & قثور الجوز & 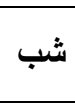 \\
\hline A & $0.1 \pm 6.39$ & $\mathrm{~A}$ & $1.4 \pm 5.5$ & - & - & 20 \\
\hline $\mathrm{A}$ & $0.11 \pm 6.45$ & $\mathrm{~A}$ & $0.35 \pm 7.25$ & - & 10 & 16 \\
\hline $\mathrm{A}$ & $0.14 \pm 6.30$ & $\mathrm{~A}$ & $0.10 \pm 6.27$ & 0.05 & - & 28 \\
\hline
\end{tabular}


و عند عكورة ماء خام 100 وحدة عكورة يظهر الجدول (7) ان استخدام الشب لوحده في التخثير اعطى اقل عكورة

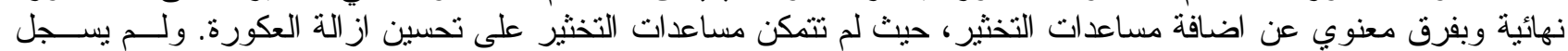

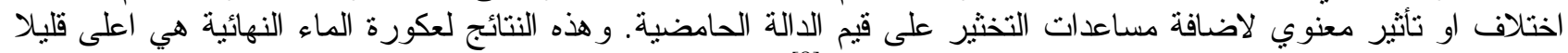

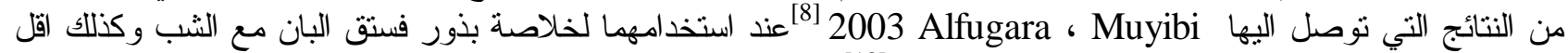

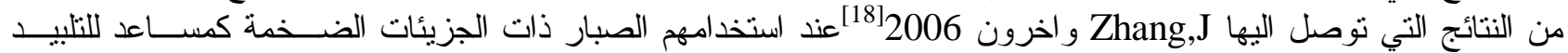

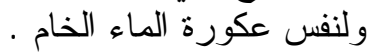

الجدول 7: العكورة النهائية والدالة الحامضية لمياه خام ذات عكورة ابتدائية 100 وحدة عكورة بعد معاملتها باستخدام الشب الثب

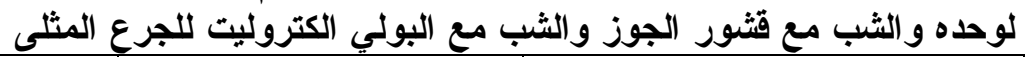

\begin{tabular}{|c|c|c|c|c|c|c|}
\hline \multirow{2}{*}{ مجموعة دنكن } & pH & محمو عة & وحدةرة النهورة & \multicolumn{3}{|c|}{ الجرعة (ملفم/تتر) } \\
\hline & المعياري الانحر اف & دنكن & المعدل الاحرراف المعياري & الكتروليتي & قالجوز & شب \\
\hline $\mathrm{A}$ & $0.11 \pm 5.455$ & $\mathrm{~A}$ & $0.04 \pm 1.33$ & - & - & 35 \\
\hline A & $0.33 \pm 5.21$ & $\mathrm{C}$ & $0.07 \pm 3.15$ & - & 200 & 30 \\
\hline $\mathrm{A}$ & $0.23 \pm 5.57$ & B & $0.07 \pm 2.15$ & 0.05 & - & 30 \\
\hline
\end{tabular}

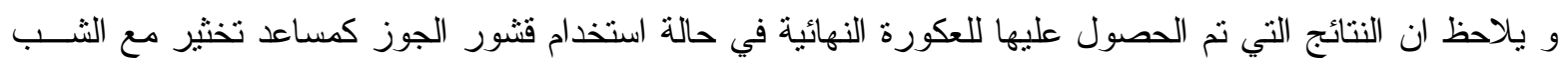

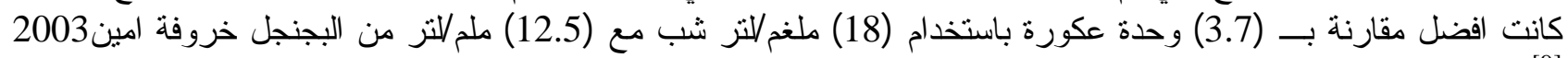

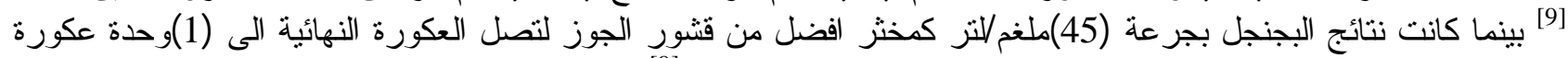

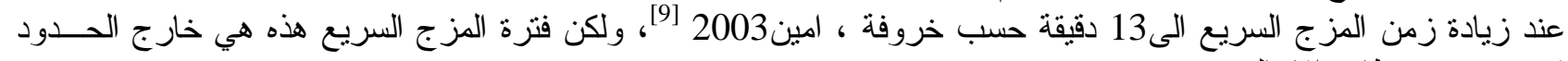
في تصميم محطات الاساله. ان الجرع العالية من قتشور الجوز ناتجة عن استخدام المادة الخام و بالامكان تخفيضها اذا اثثتت فعاليتهــا باســتخدام

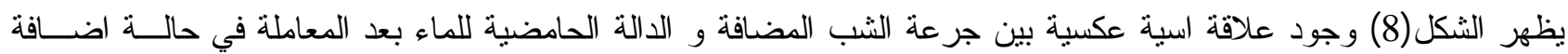

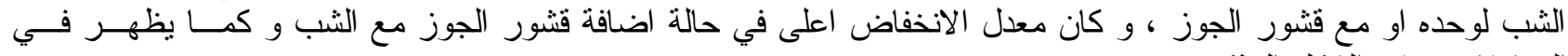
المعادلات و في الثكل المذكور .

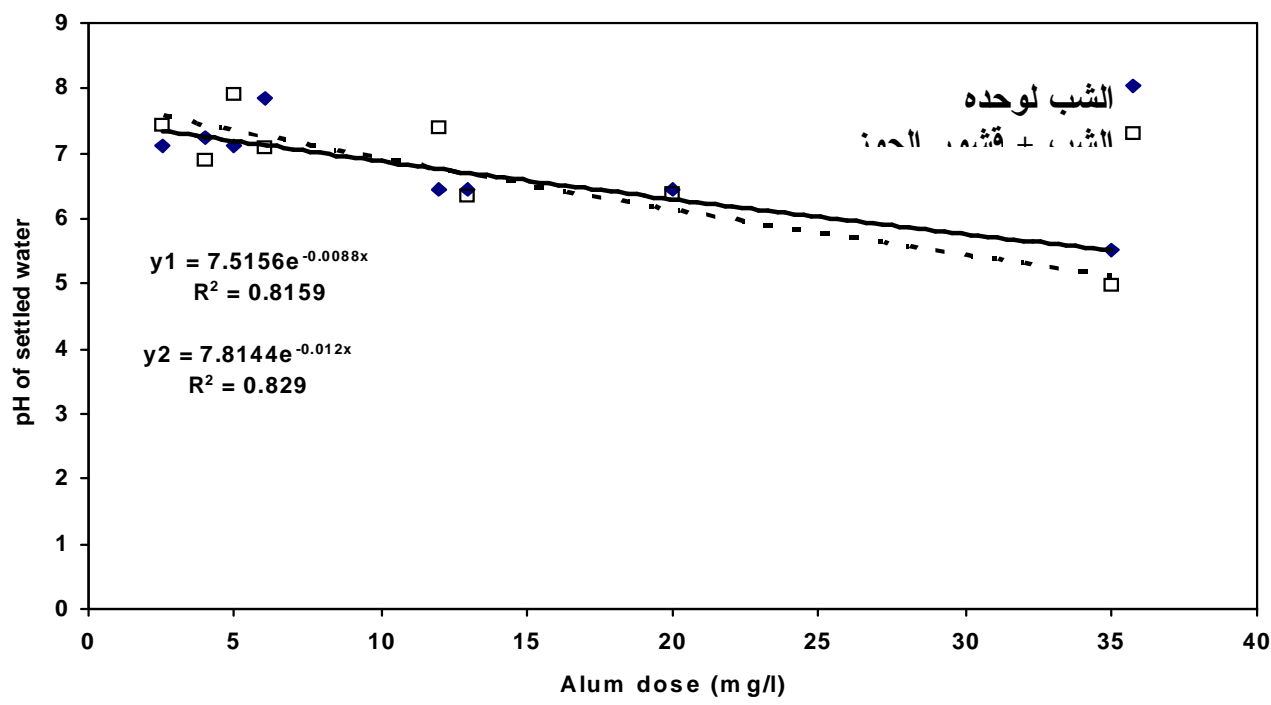

الثكل (8) تأثير استخدام قثور الجوز مع الثب على الدالة الحامضية للماء بعد الترسيب

كان أسرع تكوين للبادات في حالة اضافة مسحوق فثور الجوز كمساعد تخثير مع الثب وبعد مرور اقل من خمسـس

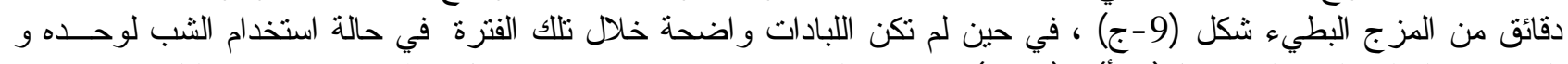

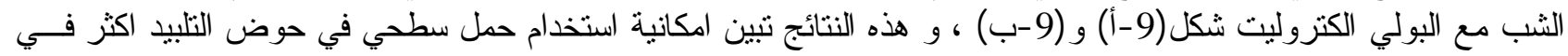
حالة استخدام قشور الجوز كمساعد للتخثير . 


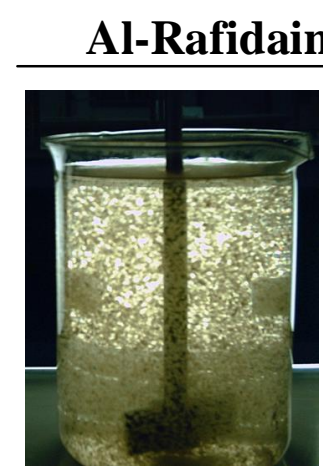

ج
Vol.17 No.3

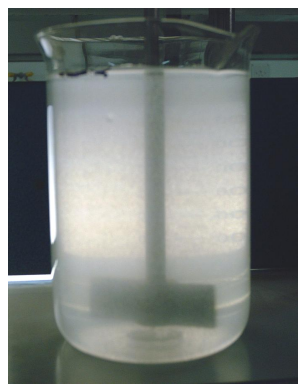

$\varphi$

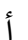

June 2009

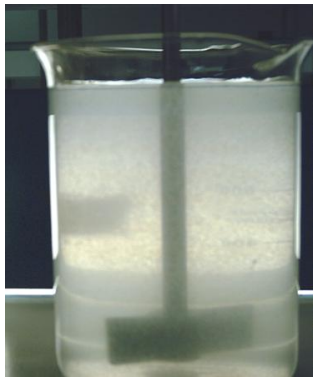

الشكل (9):صور توضح تكون اللبادات بعد مرور 5 دقائق من المزج البطيء باستخدام

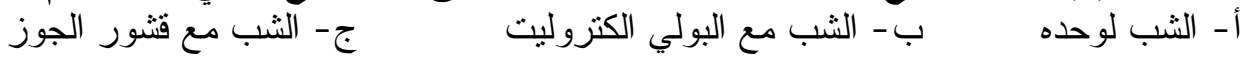

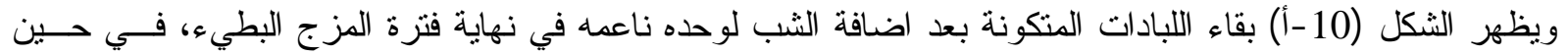

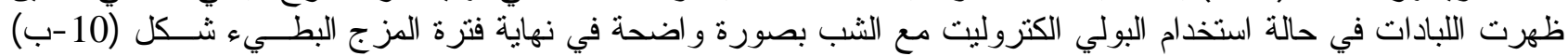

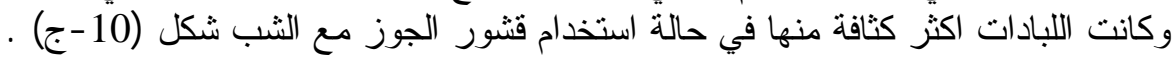

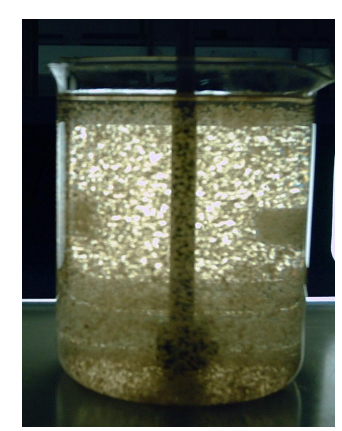

ج

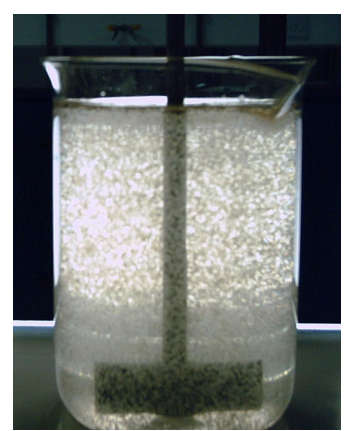

$\varphi$

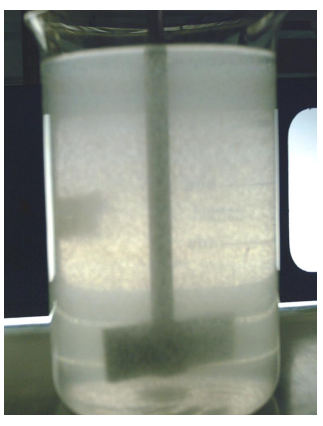

i

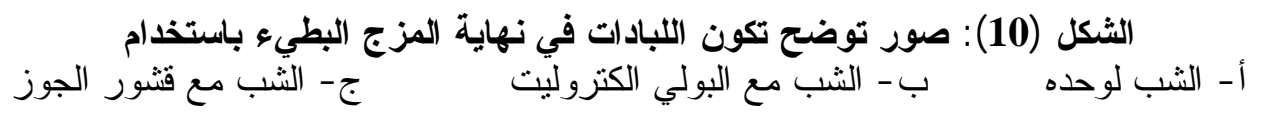

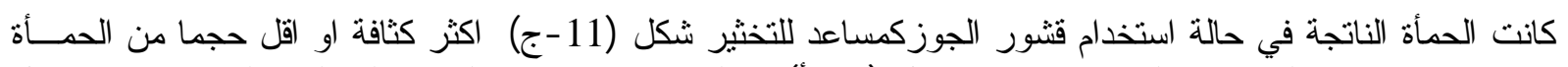

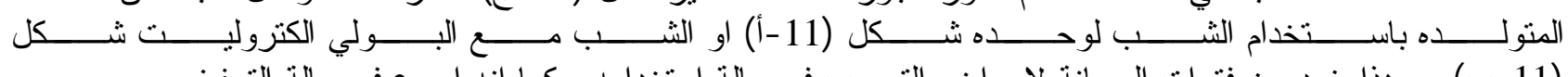

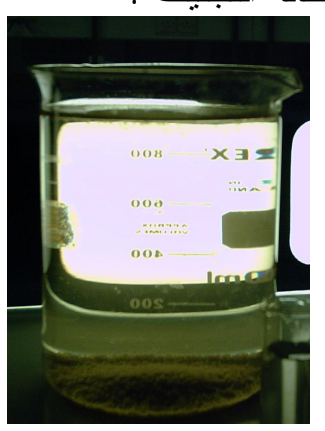

ج

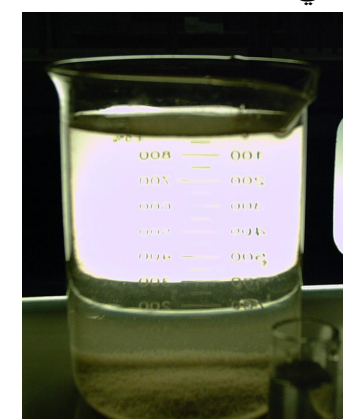

$\varphi$

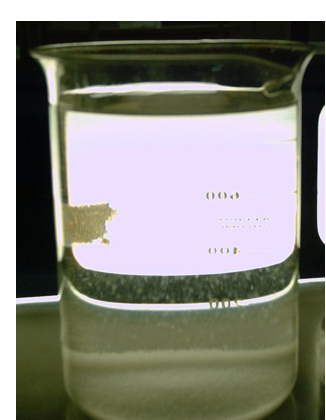

i

$$
\begin{aligned}
& \text { الشكل 11: صور توضح خصائص الحمأة المتكونة باستخدام }
\end{aligned}
$$

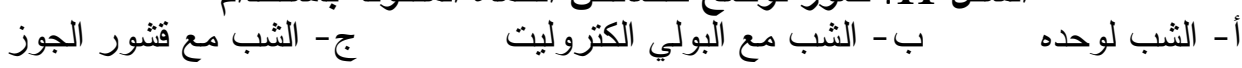


أمين :استخدام مسحوق قشور الجوز كمساعد مخثر طبيعي مع الثب بالمقارنة مع الشب لوحده أو الثب مع البولي

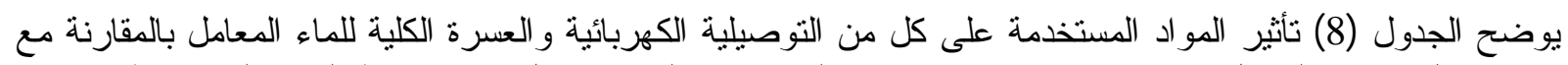
الماءالخام، يلاحظ من هذا الجدول عدم وجود نغير كبير في قيم الخصائص المذكورة قبل وبعد اضافة المو اد المستخدمة

\begin{tabular}{|c|c|c|c|c|}
\hline (ملغ//تتر) بدلالة & الكهربائية & جرع القثور & جرع الثب & عكورة الماء الخام \\
\hline كاربونات & عند(025م) & (ملغ//تتر) & (ملفم/تتر) & (وحدة عكورة) \\
\hline الكالسيوم & مايكروموز & & & \\
\hline 212 & 396 & - & - & قبل المعاملة \\
\hline 200 & 380 & 30 & 4 & 20 \\
\hline 200 & 382 & 140 & 10 & 40 \\
\hline 200 & 389 & 100 & 16 & 50 \\
\hline 200 & 393 & 10 & 16 & 80 \\
\hline 210 & 399 & 200 & 30 & 100 \\
\hline
\end{tabular}

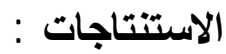

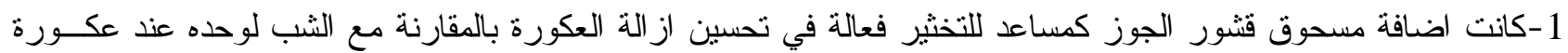

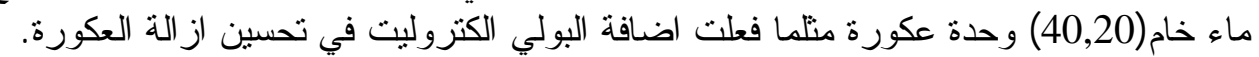

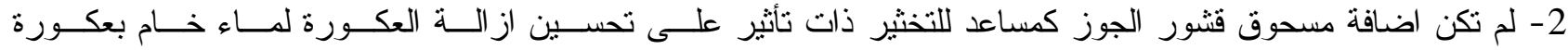

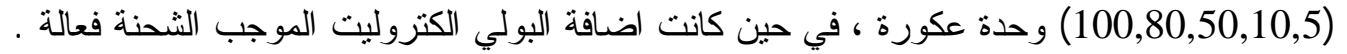

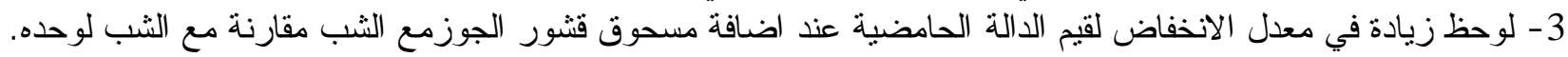

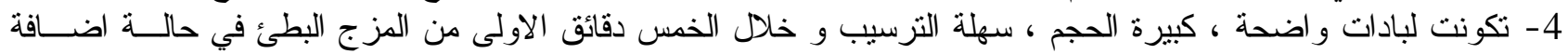

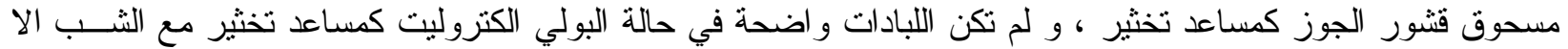

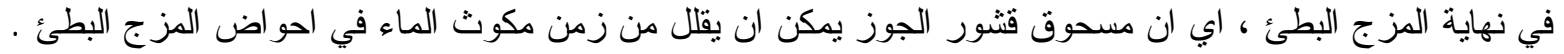

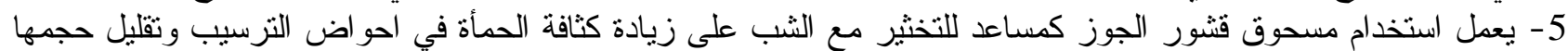

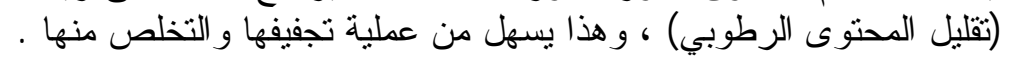

6 - لم يلاحظ وجود تغير كبير على قيم التوصيلية التونية الكهربائي و العسره الكلية للماء المعامل عند استخدام مسحوق قثنور الجوز و

الثب لمستويات العكورة المختلفة.

1. Smauel, D.F. and Osman, M.A. 1983. Chemistry of Water Treatment Copy right by Butler worth publishers.

2. Tanne, J.H. 1983, "Alzheimer and Aluminum, An Element of Suspicion", Journal of American Health, 48, Sep., USA.

3. Qureshi, N. and Malmoerg, R.H, 1985, "Reducing Aluminum Residual in Finished Water", Journal AWWA, 77: 10, Oct.

4. Al-Ani, M. Y., Hendricks, D. W., Logsdon, G. S. and Hibler, C. P. 1986, "Removing Giardia Cysts From Low Turbidity Waters by Rapid Rate Filtration", J. AWWA, 78 : 5, P. 66-73.

5. AWWA, 1997. Water treatment plant design. American Water Work Association, American Society of Civil Engineers, Third Edition, McGraw - Hill, New York.

6. Kashkuli, H.A., 1994. Coagulationchart and important notices on coagulant Usage in treatment of surface water. Seminar Proceedings of Coagulant in Water Industry, Ahvaz, Iran.

7. Letterman, R.D. and pero, R.W, 1990 Contaminant in Polyelectrolytes used in water treatment. J, AWWA., 82: 87. 


\section{$\begin{array}{llll}\text { Al-Rafidain Engineering } & \text { Vol.17 } & \text { No.3 } & \text { June } 2009\end{array}$}

8. Muyibi, s., Alfugara, A. 2003 treatment of surface water with Moringa Oleifera seed extract and alum-acomparative study using a pilot scale water treatment plant, International Journal of Environmental Studies, Volume 60, Number 6, December, pp 617-626 (10).

9. Kharrufa, S., Ameen, A.,2003. Preliminary study for the efficiency of The Carob bean on the to coagulation process, Tikrit Jour. Of Eng. Sci., Vol.10.No.1.March.P(50-65).

10. Aziz, HA; Adlan, MN; Mohamed, A M D; Raghavan, S; Isa, M K M; Abdullah, M H., 2000.Study on the anionic natural coagulant aid for heavy metals and turbidity removal in water at PH 7.5 and alum concentration $25 \mathrm{mg} / \mathrm{L}$ - laboratory scale. Indian Journal of Engineering and Materials Science (India). Vol. 7,m No.4, Aug. pp. 195-199.

11. Shehab, A.S., 1988 Effect of Using Polymer with Alum on Turbidity Removal of Tigris River Using Pilot Plant. M. Sc., Thesis, Mosul University, Engineering College.

12. Hudson, H. E., Wagner, E. G., 1981, "Conduct and use of Jar Tests", J. AWWA. 73 : 4, April, USA.

13. Al-Layla, M. A. and Middle Brooks, E. J, 1974. Optimum Values for Operational Variables in Turbidity Removal, Water and Sewage Work, No. 8, P. 66, Aug.

14. Kawamur, S. and Americas, M. W, 1996. Optimisation of Basic water Treatment Processes Design and Operation : Coagulation and Flocculation, J. Water SRT-aqua, Vol. 45, No.1, P. 3547.

15.APHA-AWWA-WPCF, 1995. Standard Methods for the Examination of Water and Wastewater, 18 Edn. American Public Health Association. Washington, D.C, U.S.A.

16.Brink,D.R.,Choi,S.,Al-Ani,M.,Hendricks,D.W.,(1988)"Bench-Scale Evaluation of Coagulants for Low Turbidity Water".Jour.AWWA80:4, April.

17.Yang,H.Y.,Cui,F.Y.,Zhao,Q.L.,Ma,Ch., 2004.Study on coagulation property of metalpolysilicate coagulants in low turbidity water treatment .Journal of Zhejiang University SCIENCE.5(6);721-726

18.Zhang, J.,Zhang, F.,Luo, Y.,Yang, H., 2006 .A preliminary study on cactus as coagulant in water treatment.Process Biochemistry, Volume. 41, Issue,March, p(730-733).

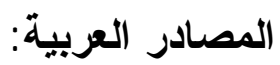

18 - اودي، بنيلوب 2002. الكامل في الأعثاب و النباتات الطبية، بأثشر اف جمعية اطباء الاعثاب في انكلترا، معجم لاتيني -

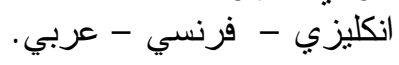

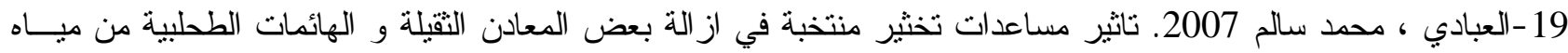

نهر دجلة . رسالة ماجستير، كلية الهندسة ، جامعة الموصل.

$$
\text { تم اجراء البحث في كلية الهندسة - جامعة الموصل }
$$

